\title{
The Analysis And The Research Of The Helicopter PIO
}

\author{
Penghui Xu' ${ }^{1}$, Yanqiu $\mathrm{Ju}^{2}$, Chi $\mathrm{Qi}^{2}$, Yue Wang ${ }^{2}$ \\ 1,2.Department of Helicopter Avionics and Weapon Engineering, Army Aviation Institute, Beijing 101123,China
}

3.The UAVS Research Center of the Training Department, Army Aviation Institute, Beijing 101123, China

Keywords: pilot induced coupling; flight control system; the stability analysis.

\begin{abstract}
Closed-loop analysis method is proposed for pilot induced coupling with helicopter flight control system in closed loop. Combining with the longitudinal identification model of a certain helicopter, McRuer pilot model, designed stability augmentation system and frequency domain model are established for pilot induced coupling analysis. By combining the basic analytical methods of the classical automatic control theory and the actual backgrounds, the properties of the root locus, the characteristics of the time domain and the frequency domain are studied. The properties as well as the reasons of the PIO are also analyzed in comparison. Meanwhile, feasible measures to improve the PIO are discussed considering the controller design and pilot's manipulation. Results show that the analysis method proposed can not only reveal the physical nature of PIO qualitatively, but also quantitatively. The analysis has theoretical reference significance in both flight control system design and pilot's manipulation applications. In the designing process of modern helicopter, high gain as well as broad band FCS has been an important target of the advantaged helicopter
\end{abstract}

designing in order to get the better handling quality. However, while the pilot and the FCS are in the control circuit, when the pilot is in the closed loop and the effect it causes must be concerned in the design of the FCS, or the quality and characters of the helicopter closed loop system will be weaken, even to cause the RPC oscillation. It may give a threat to the safety of flight.

In order to analyze the effect of the pilot in the operational circuit, in the 1940s, the academic field has started to research the pilot modeling, which is relatively mature now. For example, McRuler came up with the Crossover Model, this model shows that the open -loop total transfer function of the situation when pilot is in the handing circuit with the frequency characteristics of $H(s)=\omega_{c} \cdot e^{-\tau_{e} s} / s$ (1)

( $\omega_{c}$ is the crossover frequency, $\tau_{e}$ is the equivalent lag time). The pilot guarantees the stability of the closed loop operation by adjusting the gain to make the $\omega_{c}$ to the highest cut-off frequency. This model was proved to be an effective model, which is widely used in the analysis of the RPC and PIO. Hess came up with the Structural Model, which uses the visual perception, perception of neuromuscular system as well as vestibular system perception to be the feedback signal, describing the man-machine interaction move of double closed feedback active operation of the pilot. Mayo built the Biomechanics of feed into the model, the model shows the interactive transfer behavior of the RPC, that means the oscillation of the helicopter is transferred to the pilot by the joystick in a passive way, then the pilot makes a feedback with the stick to the helicopter by the form of feed into the biological mechanics, this model is actually a passive handling model. In common cases, the oscillation caused by the active control of the pilot belongs to the low frequency field, which is about $1-2 \mathrm{~Hz}$; but the passive control belongs to the high frequency field, which is about $2-8 \mathrm{~Hz}^{[2]}$. 
To the research of the RPC problem of the helicopter, there is an analysis of the non-steady phenomenon in vertical cone mode caused by the passive control of the pilot based on the Biomechanics of feed into the model in ${ }^{[9]}$. A combination of the Mcruler Crossover Model and the Mayo Biomechanics of feed into the model based on [9] to research the time domain analysis of vertical oscillation of the helicopter is made in ${ }^{[10]}$ and ${ }^{[11]}$, meanwhile it compares the characteristics of the Nyquist curve in frequency domain, and assures the effective of the compound pilot model which is built by himself. However, the actual FCS of the helicopter is not involved in the closed loop models in [9]-[11]. Hess analyzed different examples of RPC oscillation based on the Pilot-Structure Model in different flight tasks such as deck landing, hover, accelerating, decelerating and so on. The analysis focuses on the time domain simulation, lacking of enough discussion about the inner mechanism of the RPC oscillation. The pilot project models of different helicopter flight tasks are built and the conversion method which is equivalent to the Mcruler Crossover Model is came up with in ${ }^{[16]}$. The pilot-induced-oscillation problems caused by the static instability telex aircraft actuator rate limit and pilot model to be transferred into the simple gain link in the model are analyzed in ${ }^{[17]}$, it also discusses the effect caused by the pilot control gain as well as the actuator rate limit to the closed-loop stability.

There are mainly analysis of combining different pilot models with the helicopter dynamics models to build the RPC closed loop in the researches of the helicopter RPC oscillation problems on the

articles above. However, the FCS is barely involved in the closed-loop models in the articles. McRuler thinks that "the main reason of the RPC is caused by the failure in the design of the FCS.” So it is one-sided to discuss the opinion of the pilot-induced-oscillation solely without the FCS. Although there involves the FCS in the Hess modeling, it is so difficult to reveal the essential of the RPC directly only by using time domain analysis. Based on the above, there is a research about the essential of the RPC and the robustness of the RPC closed loop by using frequency domain analysis and root locus analysis, combining the two main factors that are pilot and FCS, which mainly cause the RPC in this article.

\section{The RPC model of the helicopter}

In order to simplify the essential of the problem, there is a simplification of the RPC model in this article as follows:

(1)The helicopter single channel model without considering the couples between the channels is used. In order to guarantee the reality of the simulation to the best, the longitudinal ferreting model of a certain helicopter is cited to be the object of study;

(2) Suppose that the flight task of the pilot is close to hover horizontal posture to keep without considering the operation of other channels as well as the effect;

(3)Suppose that the stability augmentation mode of operation is set when helicopters hover, the control response type of the longitudinal chanmel is RCAH(Rate Command Attitude Hold) mode.

The structure diagram of the closed-loop RPC of the helicopter is showed as follows in Figure 1. In the diagram ,the inner loop of the FCS is Gin ,the controller mode is $\mathrm{C}$, it is a kind of P-mode controller and it uses the roll angle $\theta$ to be the feedback signal; the helicopter model is G; the pilot adjusts the strategy of operation according to the attitude keeping task r; the control input $\mathrm{u}$ is the output of the stability augmentation operation of the total of pilot control instructions and the negative feedback input, meanwhile $\mathrm{d}$ is the external disturbances (such as the gust and so on ) to the helicopter.

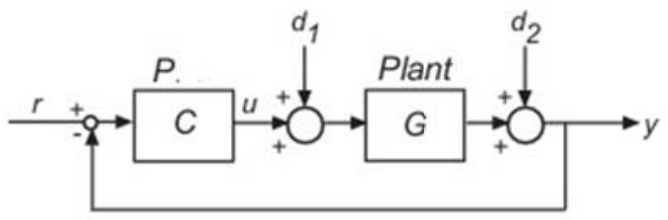

Fig. 1 Closed loop structure of RPC

The helicopter modeling, the pilot modeling and the FCS modeling are mainly concerned in the modeling of the closed-loop. Next part is the analysis on the modeling of each link in detail.

\subsection{The Helicopter model}

According to ${ }^{[18]}$, the transfer function of the longitudinal 


\section{$\frac{\theta}{\Delta B}=\frac{-(173928027 * \mathrm{~s}+2579047.8)}{16302685 * s^{3}+9708863.5 * s^{2}+169782.9 * s+617232.7} \frac{(\mathrm{rad})}{(\%)}$}

channel of the helicopter is: The identified model can reflect not only the direct correspondence of the body roll angle $\theta$ and the driving rod longitudinal manipulated variable (percentage) $\Delta B$, but also the specific mode characteristics of the helicopter. There exists the plural zero, it is so nearly to the plural pole which is correspondent to the pendulum vibration response-control input pair, it reflects the dynamic key characteristics of the coupled waving the shimmy- body system. The equivalent time delay means the control system for hydraulic and transmission delay which are known.

\subsection{The pilot model}

As a result of the conciseness and matureness of the Mcruler pilot model, and the physical meaning is conscious, this model is applied to be the RPC-pilot model. The transfer function is as follows:

$$
G_{p t}=k_{p l} \cdot \frac{T_{L} s+1}{\left(T_{l} s+1\right)\left(T_{N} s+1\right)} \cdot e^{-\tau_{p l} s}
$$

$\mathrm{S}$ is the Laplace operator; ${ }^{\tau}{ }_{p l}$ is the time delay which is correspondent to nerve conduction and motivation, it is usually about $0.13-0.30 ; 1 /\left(T_{N} S+1\right)$ is the neuromuscular delay equivalent, it means that the pilot control rate is less than the one of the pole, which is usually $0.10 ;{ }^{k}$ is pilot control gain, it is the parameter which is needed to be adjusted to guarantee the best manipulation, it is usually about $0-100 ; T_{L}$ is the lead compensation time constant which is needed in the forecast to the process of the operation by the pilot( $T_{L} \neq T_{N}$ ), it reflects the value of the pilot mental load, it is usually $0-0.25 ; T_{l}$ is the time delay of the transfer of the central information and course of working, it reflects the value of the physical strength load of
(3)

pilot, usually $0-20 ; \quad k_{p l} \bullet\left(T_{L} s+1\right) /\left(T_{l} s+1\right)$ is the adjustable parameter part of the model, which is amount to an advanced-lag link and will be adjusted by the pilot in flight. The closed-loop bandwidth when the pilot is in the circuit is usually less than $4 \mathrm{rad} / \mathrm{s}$, so if there exits an unstable mode frequency which is higher than this value, it is very difficult for the pilot to take corrective actions.

\subsection{The FCS model}

In order to build a whole closed-loop system and guarantee that the rationality of the controller design, a flight control stability augmentation system is designed in this article, the control structure is as follows:

$$
u=\frac{k_{p}\left(k_{d} s+1\right)}{T_{i} s+1} \cdot \Delta \theta=K_{\theta} \cdot \Delta \theta
$$

In the equation above, the normal parameter of the controllers is: $k_{p}=-0.27838$. The control responses of the inner stabilization loop Gin are showed in Figure 2. It can be concluded that the overshoot is $191 \%$, the steady state error is 0.5323 from the step response of the system, which indicates that the overshoot and the steady state error are relatively large, and there exists relatively violent oscillation in the initial stage of the system; However it also can be concluded that although the system is finally towards stability, it should be adjusted and improved from the respects of the dynamic as well as the steady state response according to the root locus figure and the frequency domain characteristics. The conclusion indicates that the system can't be ideally stable with the P-mode controller. So in order to have a good balance with the steady state characteristics and the dynamic characteristics, the FCS is also subject to be improved, so that the contradiction between the two aspects above can be improved, finally the whole performance of the system can be more optimized. 


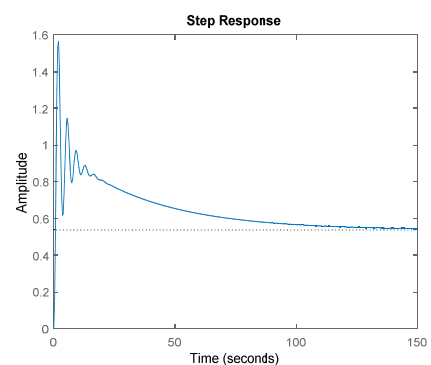

(1)

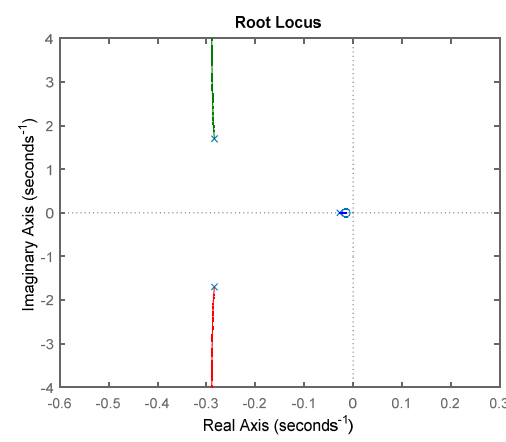

(2)

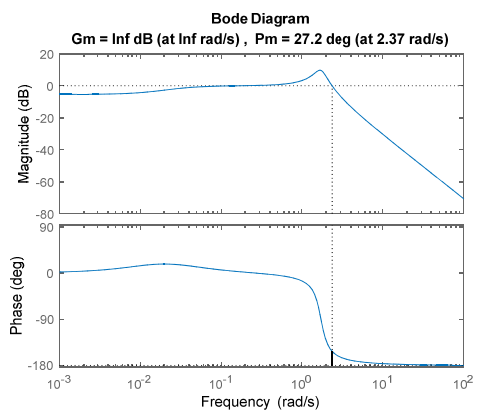

(3)

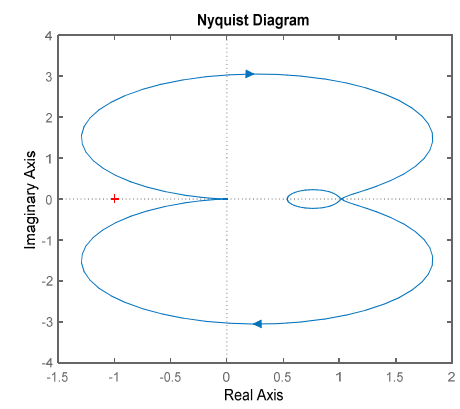

(4)

Fig. 2 Control responses of Gin

\section{The analysis of the reasons of the PIO}

The responses of the helicopter with the FCS and the pilot model are showed in Figure 3. Next part are the analysis of the PIO according to the simulation with three analysis methods of the classical control theory, the time domain analysis, the root locus analysis and the frequency domain analysis. The analysis are also combined with the actual backgrounds.

\subsection{The time domain analysis}

According to the time domain analysis of the classical control theory, there are some main respects of analysis to a certain system, which are the steady state analysis and the dynamic state analysis. The steady state progress indicates the degree of repetition of the output to the input, it can reflect some information on steady state error. And the dynamic characteristics indicate more information such as damping condition and the rate of the response based on the above. The state parameter indicators in the regulating progress are focused on. In common cases, a certain automatic control system should be "steady, accuracy and fast", the parameter indicators such as the rise time $t_{r}$, the setting time $t_{s}$, the overshoot $\sigma$, the peak time $t_{p}$ and the steady state error $\Delta$ are also used to describe the steady state and the dynamic state characteristics.

In common cases, the step response signal is used to be the classical input signal of the control system to have a research and comparison of the characteristics of all kinds of control systems. The step response curve showed in Figure 3 as below shows that the divergent state happens in the initial stage of the system of the helicopter with the P-mode controller with the pilot model, that is the unsteady state, and the largest amplitude is as about 2 times as the one of the steady state. The overshoot reaches $61.5 \%$. The unsteady state is the appearance of the PIO in the time domain, which is also the reason of the oscillation. It can be concluded from the figure that the rise time of the system is $0.522 \mathrm{~s}$, the setting time is $293 \mathrm{~s}$, the oscillation starts in the initial stage of the system as a result of the participation of the pilot delay link, the amplitude decreases as the time goes by, and about 293s later the system turns to be steady gradually. Of course the oscillation will do harm to the characteristics of the system, especially when the helicopter is going to set up, the oscillation may have a threat to the life of the pilot. In addition, the setting time is so long that it can cause some dangerous accidents easily. According to ${ }^{[1]}$, 
the dynamic characteristics is usually considered as a kind of pure gain, in this case to the whole system of the helicopter, the response rate increases and the rise time as well as the setting time decreases so the dynamic characteristics are relatively ideal and optimistic, shows in Figure 3. However, the overshoot and the steady state error will inevitably increase so as to cause the unsteady state of the whole system, which is called the PIO. Besides, the figure infers that the final value of the system is 0.841 , which is not the value 1 that it should be in the normal cases. It indicates that the steady state error still exist in the system, that is to say the system can not reach the much accurate steady state.

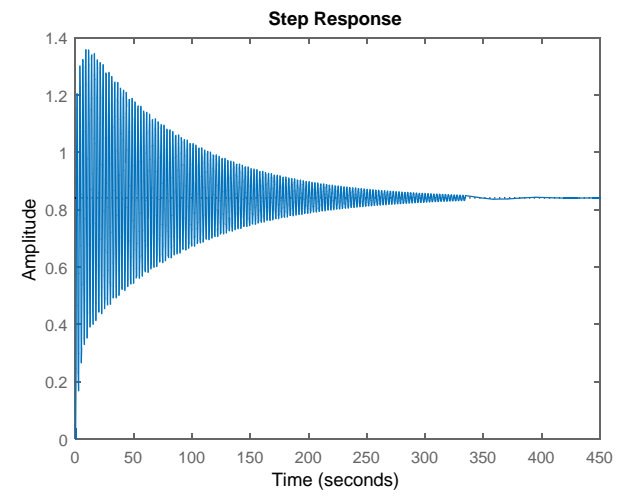

Fig. 3 Response of time domain

\subsection{The root locus analysis}

According to [2], as to the steady analysis of the gain of the system, the motion modes of the helicopter can be considered as the appearances of all kinds of integrated effects of modes, each kind of mode involves special characteristics, such as the mode frequency, damping, and the capacity of resisting disturbance and so on. So the actual motion condition of the helicopter is determined by the characteristics of these modes. The locus which changes with the parameters of the closed-loop poles can be determined by observing the root locus of the modes according to the distribution of the open-loop zeros and poles when the parameters of the systems changes. It is convenient and accurate because the effect tendency of the changes of the parameters of the system to the closed-loop poles can be concluded by simple calculation when the root locus analysis is applied. This kind of qualitative analysis values a lot in the research of the system characteristics and the reasonable methods of improving the characteristics.
Figure 4 shows us the root locus of the system with the pilot model, it can be concluded that a certain part of the root locus exists in the right of the complex plane, some closed-loop poles exists separately in the imaginary axis and the right plane. According to the Routh Criterion and the Root Locus steady state Criterion, it can be concluded that there are unstable responses in the system, and the system is unsteady. According to ${ }^{[2]}$, there are the phugoid mode and the pitch subsidence mode in the system now, and the characteristics of the phugoid will cause the decrease of the system damping, which can lead to the unsteady state easily, that is the oscillation. With the appearance of these two modes, the center of gravity of the helicopter happens to change, so do the moment of forces. It can lead to the opposite phenomenon of the short period mode. So from the aspect of the complex plane of the root locus, the reason of the PIO is also very obvious, that is the changes of the helicopter mode which causes the changes of the whole characteristics and state. Then the PIO happens.

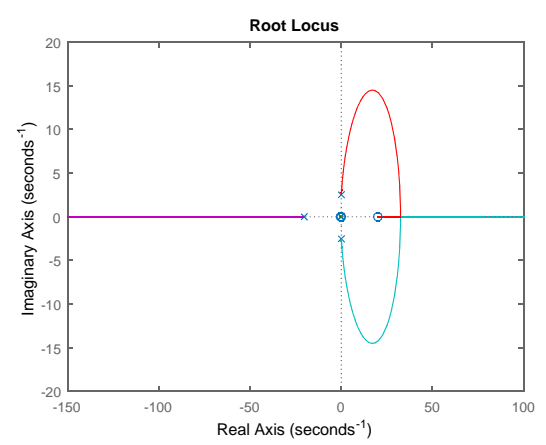

Fig.4 Rlocus mode figure

\subsection{The frequency domain analysis}

According to the frequency domain stability criterion, the pilot-rotorcraft system belongs to a kind of non-minimum-phase system. From ${ }^{[22]}$ and the Bode diagram as well as the Nyquist curve in Figure 5, it can be concluded that the phase margin of the system is -19.3deg, the magnitude margin is $-25.8 \mathrm{~dB}$, which are all negative. According to the Nyquist stability Criterion, the equation $\mathrm{R}=\mathrm{P}-\mathrm{Z}$ is not zero. So from the perspective of the frequency domain stability, the system is not stable, that is the appearance of the PIO.

According to ${ }^{[1]}$, in common cases during the flight, the reasons of the PIO are usually: 
(1) When the pilot is trying to adjust the gain of the system; (2)The pilot is trying to make a lead compensation correction in the low frequency as a result of the decreased phase when he wants to overcome the extra delay in the frequency domain of the system;

(3)The oscillation phenomenon in the low frequency which is led by the biomechanics feedback of the pilot to the stick to increase the stability of the pilot-rotorcraft system.

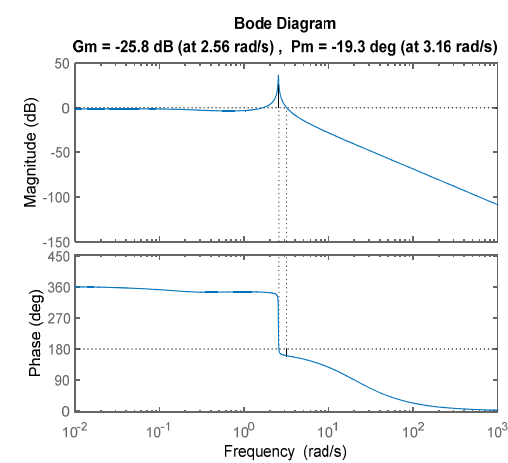

(1)

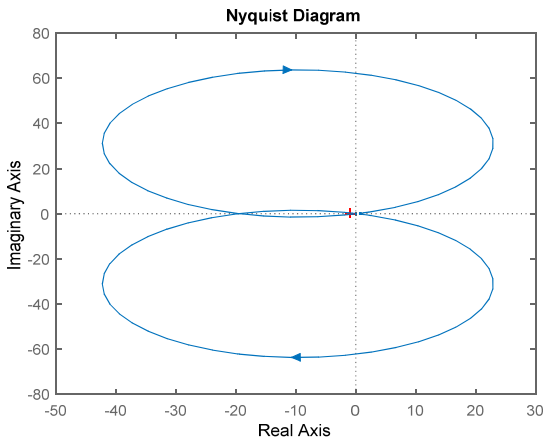

(2)

Fig.5 Response of frequency domain, Bode diagram and Nyquist Figure

There are different perspectives of the analysis on the reasons of the PIO according to the classical control theory and the actual backgrounds in the parts above, the methods of the analysis used are time domain analysis, root locus analysis, frequency domain analysis. However, it is becoming more and more important that how to take some feasible and effective measures to improve the PIO.

\section{The feasible measu res to i mprove the PIO in theory.}

Figure 6 shows us the characteristics of the improved system as below. Now the FCS is improved in a feasible way, and the pilot operation is disconnected. It can reduce the degree of the PIO and make the system stable gradually. The FCS is a kind of PID controller, the control structure is :

$u=\frac{K_{p}\left(K_{d 1} s+1\right)\left(K_{d 2} s+1\right)}{\mathrm{T}_{\mathrm{i}} s+1} \Delta \theta=K_{\theta} \cdot \Delta \theta$

the parameters are: $K_{p}=-0.20665, K_{d 1}=0.97$, $K_{d 2}=1.70, T_{i}=0.025$. It also can be concluded from Figure 7 that the root locus and the closed-loop poles are all in the left side of the complex plane. From the frequency domain, the phase margin and the magnitude margin are positive. According to the Nyquist stability Criterion, the system is finally stable. It can be inferred that the overshoot is $15.8 \%$, the rise time is $0.354 \mathrm{~s}$, the setting time is $2.44 \mathrm{~s}$, the steady state error is 0 from the step response of the improved system. It indicates that the both the dynamic and the steady characteristics of the system are coordinated well, and the capacity of resisting disturbance is relatively better. Now the system is fit for the actual need and the measure is feasible in theory.

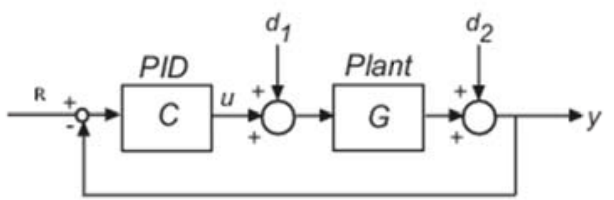

(1)

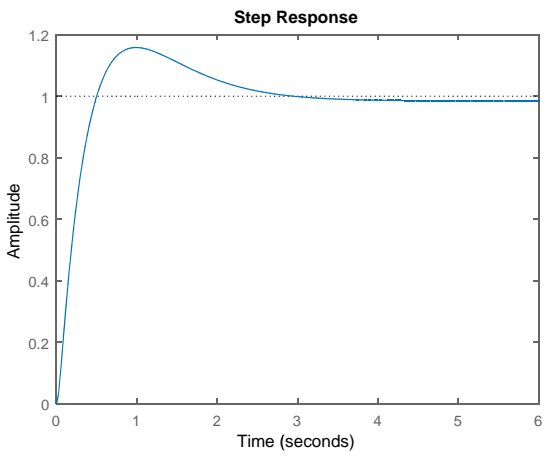

(2) 


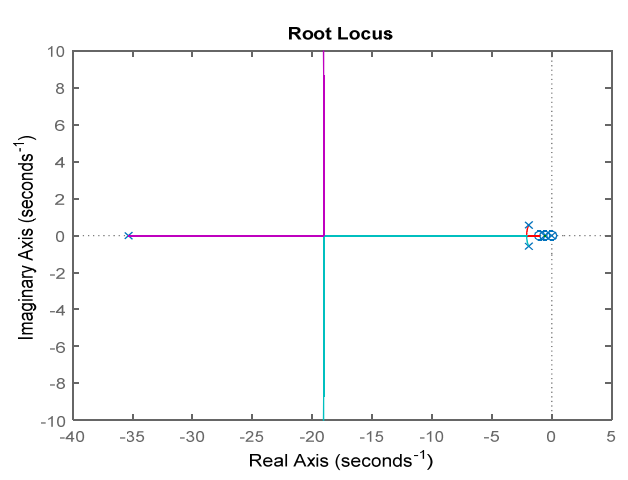

(3)

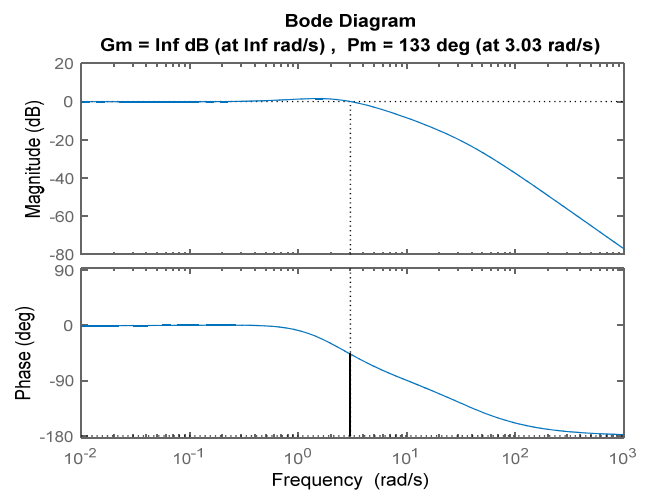

(4)

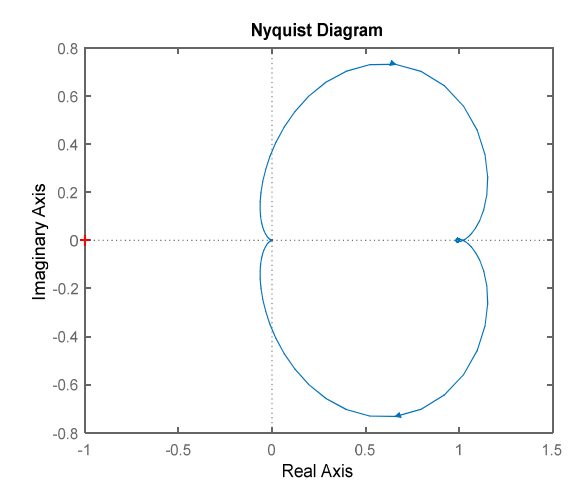

(5)

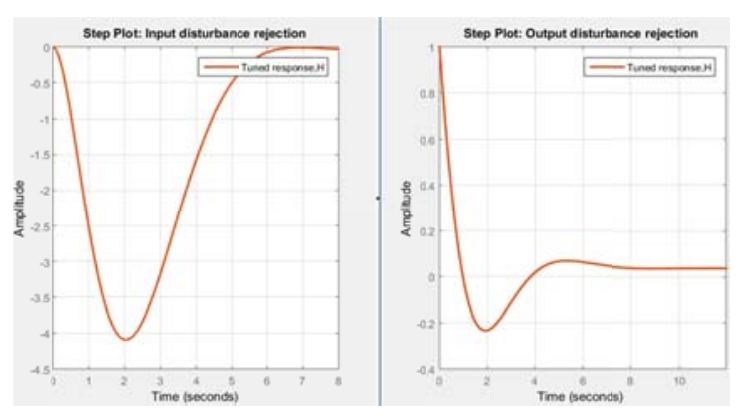

(6)

Fig. 6 Response and figure of improved system

\section{Conclusions}

This article has a discussion about some common reasons about the PIO and gives some analysis with the classical control theory, also there are some feasible measures and ideas involved to improve the problem according to the result of the simulation. The conclusions are clear based on the simulation and the analysis above:

(1) During the actual flight, the PIO is caused as a result of the handing quality of the pilot and the changes of the compliance of the helicopter, which can be analyzed in the time domain, frequency domain as well as the toot locus in classical control theory. The reason can be concluded quantitively and qualitatively.

(2)The result of the simulation indicates that by improving the FCS and disconnecting the pilot model, the PIO can be effectively adjusted to some extent and the pilot-helicopter system can keep a relatively steady state. In this article, the PID-mode controller is chosen as the improved FCS.

(3) The essential reasons of the PIO can be indicated with the actual backgrounds and the representative pilot model, it can give persuasive ideas, which values a lot in providing some theoretic ideas and measures.

\section{Acknowledgements}

The author gratefully acknowledges the contributions of the director Junfeng Chen, who provides some important ideas and data for this article. The author has received much help from the 2nd and the 3rd author in the research.

\section{References}

[1] McRuer, D. T.. Aviation Safety and Pilot Control: Understanding and Preventing Unfavourable Pilot-Vehicle Interactions[M]. Washington, DC: National Academy Press, (1997).

[2] Marilena D. Pavel, Michael Jump, BinhDang Vu, et al. Adverse rotorcraft pilot couplings-Past, present and future challenges[J]. Progress in Aerospace Sciences, 62(1): pp.1-51,(2013).

[3] Tustin A. The nature of the human operator's response in manual control and its implications for controlled design[J]. 
Journal of IEE, 94(1): pp. 190-202, (1947).

[4] McRuer, D. T., Jex, H. R.. A Review of Quasi-Linear

Pilot Models[J]. IEEE Transactions on Human Factors in Electronics,8(3): pp.231-249, (1967).

[5] McRuer, D. T., Krendel, E. S.. Mathematical Models of Human Pilot Behavior[R]. AGARD AG-188, (1974).

[6] Hess, R. A.. Theory for Aircraft Handling Qualities Based Upon a Structural Pilot Model[J]. Journal of Guidance, Control, and Dynamics, 12(6): pp.792-797, (1989).

[7] Hess, R. A.. Simplified Approach for Modelling Pilot Pursuit Control Behaviour in Multi-Loop Flight Control Tasks[J]. Journal of Aerospace Engineering, 220(2): pp.85-102, (2006).

[8] J. R. Mayo. The involuntary participation of a human pilot in a helicopter collective control loop[C]// 15th European Rotorcraft Forum, Amsterdam, The Netherlands: pp.1-12, (1989).

[9] Giuseppe Quaranta, Pierangelo Masarati, Joost Venrooij. Impact of pilots' biodynamic feedthrough on rotorcraft by robust stability[J]. Journal of Sound and Vibration, 332(1): pp.4948-4962, (2013).

[10] Pierangelo Masarati, Giuseppe Quaranta, Andrea Bernardini et al.. Voluntary Pilot Action Through Biodynamics for Helicopter Flight Dynamics Simulation[J]. Journal of Guidance, Control, and Dynamics, 38(3): pp. 431-441, (2015).

[11] Vincenzo Muscarello, Giuseppe Quaranta, Pierangelo Masarati. The role of rotor coning in helicopter proneness to collective bounce[J]. Aerospace Science and Technology, 36(1): pp.103-113, (2014).

[12] Hess, R. A.. Modeling Human Pilot Adaptation to Flight Control Anomalies and Changing Task Demands[J]. Journal of Guidance, Control, and Dynamics, Article in Advance, 6(1): pp.1-12, (2015).

[13] Hess, R. A.. Modeling the Pilot Detection of Time-Varying Aircraft Dynamics[J]. Journal of Aircraft, 49(6): pp.2100-2104, (2012).

[14] Hess, R. A., Federico Marchesi. Analytical Assessment of Flight Simulator Fidelity Using Pilot Models[J]. Journal of Guidance, Control, and Dynamics, 32 (3): pp. 760-770, (2009).

[15] Hess, R. A.. Simplified Technique for Modeling
Piloted Rotorcraft Operations Near Ships[J]. Journal of Guidance, Control, and Dynamics, 29(6): pp. 1339-1349, (2006).

[16] Songshan YANG. Research on Helicopter Pilot Model[J]. Flight Dynamics, 11(4): pp.42-47, (1993).

[17] Qimeng CAO, Yinghui LI, Haojun XU. Stability region of Closed-loop Pilot-vehicle System for Fly-by-wire Aircraft Based on Linear Matrix Inequalities[J]. Acta Aeronautica et Astronautica Sinica, 34(1): pp.19-27, (2013). [18] Tischler, M. B.. System identification requirements for high-bandwidth rotorcraft flight control system design[J]. Journal of Guidance, Control, and Dynamics, 13(5): pp.835-841, (1990).

[19] Kemin Zhou, Jone C. Doyle, Keith Glover. Robust and Optimal Control[M]. Jianqin Mao, Yisheng Zhong, translated. Beijing: National Defence Industry Press: pp.322-326(in Chinese), (2006).

[20] US Army AMCOM. Aeronautical design standard ADS33E-PRF, performance specification, handling qualities requirements for military rotorcraft[S]. Redstone, Alabama: ASMC N/A: pp.76-77, (2000).

[21] Robert T. N. Chen, William S Hindson. Influence of High-Order Dynamics on Helicopter Flight-Control System Bandwidth[J]. Journal of Guidance, Control, and Dynamics, 9(2): pp.190-197, (1986).

[22] Xinliang Zheng, The research of the characteristics of the linear non-minimum-phase system[J](2), The industry techniques of Shandong. (2015). 